\title{
ADSORÇÃO E FORMAS DE FÓSFORO EM LATOSSOLOS: INFLUÊ NCIA DA MINERALOGIA E HISTÓRICO DE USO(1)
}

\author{
P. E. F. MOTTA ${ }^{(2)}$, N. CURI (3), J . O. SIQUEIRA(3), B. VAN RAIJ (4), \\ A. E. FURTINI NETO(5) \& J . M. LIMA ${ }^{(5)}$
}

\begin{abstract}
RESUMO
Neste trabalho, avaliou-se a influência da mineralogia e o histórico de uso na adsorção e formas de P em L atossolos. E mpregaram-se cinco Latossolos (LAx, LAd, LVw e dois LVdf, desenvolvidos de gabro, $L$ ddfg, e de tufito, LVdft), de modo a abranger uma ampla faixa de proporções de hematita, goethita, caulinita (Ct) e gibbsita (G b). E stes solos foram coletados em áreas nunca cultivadas e em áreas adjacentes já cultivadas por longos períodos, onde receberam calagens e adubações fosfatadas periódicas. Procedeu-se à caracterização física, química e mineralógica, envolvendo: granulometria, Fe em formas menos cristalinas $\left(\mathrm{Fe}_{\mathrm{o}}\right)$, óxidos livres $\left(\mathrm{Fe}_{\mathrm{d}}\right)$ e "totais" $\left(\mathrm{Fe}_{\mathrm{s}}\right)$, além do fracionamento e disponibilidade de P. Com o uso da difração de raios-x, obteve-se a composição mineralógica das frações: argila desferrificada e argila ferro-concentrada, e, por análise térmica diferencial, a razão $\mathrm{Ct} /(\mathbf{C t}+\mathbf{G b})$, na fração argila desferrificada. A adsorção de $P$ foi estudada, empregando-se 24 h de agitação e concentrações de 0; 5; 10; 15; 25; 50; 75; 100 e $200 \mathrm{mg} \mathrm{L}^{-1}$, para calcular a isoterma de adsorção, de onde se obteve a capacidade máxima de adsorção de fósforo (CMAF). Os resultados mostraram que, à medida que a mineralogia dos Latossolos tornou-se mais oxídica, aumentaram a adsorção de $\mathrm{P}$, o teor total $\mathrm{e}$ as formas ligadas mais fortemente $\mathrm{a}$ Al e Fe. $O$ cultivo influenciou, de modo diferenciado, a adsorção de $P$ e aumentou as formas de $\mathbf{P}$ ligado a $\mathrm{Ca}$ em todos os solos. As formas pouco lábeis de $\mathbf{P}$ predominaram nos Latossolos estudados, destacando-se as orgâni cas associadas a compostos húmicos naqueles não cultivados e as inorgânicas ligadas a Fe e Al nos cultivados.
\end{abstract}

Termos de indexação: disponi bilidade de fósforo, fracionamento de fósforo.

\footnotetext{
(1) Parte da Tese de Doutorado apresentada pelo primeiro autor à U niversidade Federal de Lavras - UF LA. Recebido para publicação em novembro de 2000 e aprovado em julho de 2001.

(2) Pesquisador da Embrapa Solos. Rua J ardim Botânico 1024, CEP 22460-000 Rio de J aneiro (RJ ). Bolsista do CNPq. E-mail: motta@cnps.embrapa.br

(3) Professor Titular do Departamento de Ciência do Solo, Universidade Federal de Lavras - UFLA. Caixa Postal 37, CEP 37200-000 Lavras (MG). Bolsista do CNPq. E-mail: niltcuri@ufla.br; siqueira@ufla.br

(4) Pesquisador da Embrapa Meio Ambiente. Rodovia SP 340 km 127,5, Caixa Postal 69, CEP 13820-000 Jaguariúna (SP). E-mail: bvanraij@cnpma.embrapa.br

(5) Professor Adjunto do Departamento de Ciência do Solo, UFLA. Bolsista do CNPq. E-mail: afurtini@ufla.br; jmlima@ufla.br
} 


\title{
SUMMARY: ADSORPTION AND FORMS OF PHOSPHORUS IN LATOSOLS: INFLUENCE OF MINERALOGY AND USE
}

\begin{abstract}
The aim of this study was to verify theinfluence of mineralogy and former soil use on adsorption and forms of $\mathrm{P}$ in Latosols. The following five soils were used to includea wide range of contents of hematite, goethite, kaolinite ( $\mathrm{Ka}$ ) and gibbsite (Gi): (cohesive Yellow Latosol, dystrophic Yellow Latosol, acric Red Latosol, and two dystropherric Red Latosols, one developed from gabbro and one from tuffite), collected from non-cultivated areas and adjacent areas cultivated for long periods, receiving liming and phosphated fertilization periodically. Physical, chemical and mineralogical characterizations were performed, involving particlesize distribution, $\mathrm{F}$ ein less crystalline $\left(\mathrm{Fe}_{\mathrm{o}}\right)$, fre oxides $\left(\mathrm{Fe}_{\mathrm{d}}\right)$ and "total" $\left(\mathrm{Fe}_{\mathrm{s}}\right)$ forms, besides $\mathrm{P}$ fractionation and availability. Phosphorus adsorption was studied using $24 \mathrm{~h}$ of shaking and concentrations of $0 ; 5 ; 10 ; 25 ; 50 ; 75 ; 100$ and $200 \mathrm{mg} \mathrm{L}^{-1}$, to obtain the adsorption isotherm from which the maximum adsorption capacity of $\mathrm{P}$ was obtained. The mineralogical composition of $\mathrm{Fe}$ freeand $\mathrm{Fe}$-concentrated clay fractions was obtained using $\mathrm{x}$-ray diffraction analysis and $\mathrm{Ka} /(\mathrm{Ka}+\mathrm{Gi})$ ratio in theF efreclay fraction through differential thermal analysis. As themi neralogy of the Latosols becamemoreoxidic, therewas an increase in $\mathrm{P}$ adsorption, total $\mathrm{P}$ and the forms morestrongly linked to Feand Al. Cultivation differentially influenced $P$ adsorption and increased theforms of $P$ linked to $\mathrm{Ca}$ in all thesoils. Less labileP forms predomi nated in thesoils, especially theorganic ones associated to humi c compounds in thenon-cultivated soils and to theinorganic ones linked to Fe and $\mathrm{Al}$ in the cultivated soils.
\end{abstract}

Index terms: phosphorus availability, phosphorus fractionation.

\section{NTRODUÇÃO}

Embora o P, em termos quantitativos, seja um dos macronutrientes menos exigidos pelas plantas, é o que, com mais freqüência, tem limitado a produção agrícola em condições brasileiras (Malavolta, 1976; Raij, 1991), o que se deve tanto à sua deficiência generalizada em solos tropicais quantoà sua imobilização em decorrência das fortes interações queapresenta com os constituintes destes solos. A sorção de $\mathrm{P}$, que inclui tanto adsorção na superfície de minerais quanto sua preci pitação como fosfatos de baixa solubilidade, é comum em solos ácidos, relativamente ricos em óxidos de Fe e de Al, como é o caso geral dos Latossolos.

A magnitude deste fenômeno depende da natureza e da quantidade de sítios disponíveis na superfície dos minerais, sendo, por isso, dentro de uma mesma mineralogia, afetada pelo maior teor de argila (Leal \& Veloso, 1973; Bahia Filho et al., 1983). Assim, em sol os deficientes em $P$ e com grande quantidade de argilominerais e óxidos, a adsorção de $P$ é maior e, para o atendimento da exigência das culturas, são exigidos níveis de adubação fosfatada mais el evados do que para sol os mais arenosos.

Numerosos trabal hos têm mostrado, entretanto, que, além da quantidade de argila, sua constituição mineralógica é de suma importância na adsorção de P pelos sol os, o que se justifica pela peculiaridade da energia de ligação entre o $\mathrm{P}$ e cada fração adsorvente.
Nesse aspecto, os óxidos de Fe e de Al são tidos como os constituintes da fração argila mais efetivos na adsorção de $P$ (Parfitt, 1979), sendo a goethita considerada o principal componente da fração argila responsável por este fenômeno em solos do Brasil Central (Bahia Filhoet al., 1983). A maior capacidade de adsorção de $\mathrm{P}$ de sol os goethíticos em relação aos hematíticos foi também constatada por Bigham et al. (1978), Curi \& Franzmeier (1984) e Peña \& Torrent (1984), e pode ser creditada, segundo Frossard et al. (1994), às diferenças quanto à facilidade de acesso dos fosfatos a grupos $\mathrm{OH}^{-}$de superfície. Por afetar a extensão da superfície reativa do mineral, a morfologia dos cristais dos óxidos de ferro influencia também a adsorção de P (Torrent et al., 1990; Ruan \& Gilkes, 1996).

A participação dos óxidos de Al neste fenômeno tem sido, de modo geral, menos destacada, apesar de a gi bbsita ter participação importantena adsorção de $P$, mesmo que de forma menos efetiva que a goethita (McLaughlin et al., 1981). Em razão da sua ocorrência em grandes quantidades em alguns Latossol os argil osos muito vel hos, sua contribuição, em termos de adsorção total, pode, todavia, ultrapassar a dos óxidos de ferro (Curi et al., 1988; Mesquita Filho\& Torrent, 1993). O papel da caulinita na adsorção de $P$ parece secundário em comparação com os componentes oxídicos. Ker (1995) observou que o aumento do caráter caulinítico em diversos Latossolos do Brasil ocasionava a redução da capacidade de adsorção de P. 
O P apresenta-se no solo sob diferentes formas, tendo sido propostos vários métodos químicos, baseados em extrações seqüenciais, para o seu fracionamento. O método de Chang \& J ackson (1957) distingue principalmente as frações P-Al, P-Fe e P-Ca, enquanto o de Hedley et al. (1982) separa as formas lábeis, pouco lábeis e não-lábeis. A maioria dos trabalhos nesta linha tem se concentrado na quantificação das formas de P sem, no entanto, associá-las à mineralogia.

A remoção de $P$ pelas plantas e as alterações na sua disponibilidade pela calagem e adubações fosfatadas ou orgânicas alteram as formas deste elemento no solo, sendo, porém, raros os trabal hos que procuram esclarecer as formas preferenciais para onde migra o $\mathrm{P}$ adicionado e de quais destas formas provém o $\mathrm{P}$ absorvido pelas plantas. Por outro lado, a el evada potencialidade agrícola e a vasta extensão de ocorrência dos Latossolos no Brasil justificam estudos básicos para obtenção de dados que auxiliarão no fornecimento de soluções mais adequadas aos casos específicos, visando ao seu uso e manejo sustentável.

O objetivo destetrabalho foi verificar a influência da mineralogia e histórico de uso na adsorção e formas de $\mathrm{P}$ em alguns dos principais Latossolos brasileiros.

\section{MATERIAL E MÉTODOS}

\section{Solos e amostragem}

Para abranger ampla variação nas proporções de hematita, goethita, caulinita e gibbsita, foram utilizados solos argilosos e muito argilosos, classificados no Sistema Brasileiro de Classificação de Solos (EMBRAPA, 1999) como: (a) Latossolo Amarelo coeso ( $L A X)$, solo desenvolvido de sedimentos argilo-arenosos do Grupo Barreiras, do Terciário, e caracterizado pela predominância na fração argila de minerais 1:1 do grupo da caulinita; (b) Latossolo Amarelo distrófico (LAd), desenvolvido do produto da decomposição in situ de gnaisses; (c) Latossolo Vermelho ácrico (LVw), desenvolvido de sedimentos finos do Terciário que recobrem as chapadas do Brasil Central, e em cuja fração argila predominam óxidos de $\mathrm{Fe}$ e de $\mathrm{Al}$; (d) Latossolo Vermel ho distr oférrico, derivado de gabro (LVdfg), e (e) Latossolo Vermelho distroférrico, derivado de tufito (LVdft), rocha básica não endurecida composta de mistura de detritos piroclásticos e cinzas vulcânicas, com altos teores de $\mathrm{Fe}, \mathrm{Ca}, \mathrm{K}$ e P (Guimarães, 1955).

Estes sol os foram col etados sob vegetação nativa e em área adjacente, cultivada por longos períodos, quando receberam cal agens e adubações fosfatadas periódicas. A profundidade de coleta foi fixada em
0-20 cm. Visando dispor de pares de solos com atributos físicos e mineralógicos semelhantes, mas com diferentes disponibilidades de $\mathrm{P}$, os pontos de coleta, dentro de cada classe de solo, distaram de, no máximo, 200 m (Quadro 1). O material coletado foi seco ao ar, passado em peneira de $2 \mathrm{~mm}$ e submetido às análises físicas, químicas e mineralógicas.

\section{Métodos de laboratório}

A granulometria foi determinada pel o método da pipeta, utilizando-se $\mathrm{NaOH} 0,1 \mathrm{~mol} \mathrm{~L}^{-1}$, como dispersante, e agitação lenta (4 h), para a máxima preservação dos cristais dos minerais, com vistas no aproveitamento posterior da fração argila para as análises mineralógicas. Foram efetuadas cinco repetições.

As análises químicas de rotina, realizadas de acordo com EMBRAPA (1997), incluíram a determinação do $\mathrm{pH}$ em água, complexo sortivo, $\mathrm{P}$ disponível (Mehlich-1), micronutrientes eC orgânico, perfazendo três repetições (Quadro 2). Na argila, determinou-se o ferro extraído por oxalato deamônio ácido, conforme Schwertmann (1964) (uma extração), e ditionito-citrato-bicarbonato de sódio (DCB) (quatro extrações sucessivas), conforme Mehra \& J ackson (1960). Os óxidos do ataque sulfúrico foram determinados, conforme Vettori (1969), com modificações (E MBRAPA, 1997).

A adsorção de $P$ foi estudada, conforme Olsen \& Watanabe (1957), empregando-se 24 h de agitação, 2 g deTFSA, corrigida posteriormente para TFSE, e agitação horizontal com $40 \mathrm{~mL}$ de solução de $\mathrm{CaCl}_{2}$ $0,01 \mathrm{~mol} \mathrm{~L}^{-1}$ com diferentes concentrações de fósforo (0; 5; 10; 15; 25; 50; 75; 100 e $200 \mathrm{mg} \mathrm{L}^{-1}$ ). O P não detectado no sobrenadante após centrifugação foi considerado como adsorvido. Os dados obtidos em três repetições foram ajustados à equação linear da isoterma de Langmuir, $C / x / m=1 / a b+C / b$, em queC éa concentração de $P$, em $\mathrm{mg} \mathrm{L}^{-1}$, na solução de equilíbrio, $x /$ méa razão entre a massa de $\mathrm{P}$ adsorvido e a massa de solo, em $\mathrm{mg} \mathrm{kg}^{-1}$, a uma constante relacionada com a energia de retenção dos íons e b a adsorção máxima de P.

A mineralogia da argila foi determinada por difração de raios-X (DRX), utilizando lâminas de sedimentação. Esta fração foi também submetida ao tratamento com NaOH $5 \mathrm{~mol} \mathrm{~L}^{-1}$ fervente (Kämpf \& Schwertmann, 1982), para concentração dos óxidos de $\mathrm{Fe}$, sendo o material resultante montado em suportes de alumínio e submetido também à DRX, para identificação e caracterização dos óxidos deFe.

Com base nos difratogramas, foi determinada a proporção de hematita egoethita, por meio da razão entre as intensidades dos reflexos 012 da hematita e 110 da goethita, conforme Kämpf \& Schwertmann (1982), e a substituição em Al na estrutura da goethita, segundo Schulze (1984). A quantificação de caulinita e gibbsita foi feita por análise térmica 


\section{Quadro 1. Informações básicas sobre os solos estudados}

\begin{tabular}{|c|c|c|c|c|c|}
\hline Solo & $\begin{array}{c}\text { Material de } \\
\text { origem }\end{array}$ & $\begin{array}{c}\text { Vegetação } \\
\text { nativa }\end{array}$ & Localização & Uso & Manejo \\
\hline $\begin{array}{l}\text { Latossolo Amarelo } \\
\text { coeso (LAx) }\end{array}$ & $\begin{array}{l}\text { Sedimentos } \\
\text { da Grupo } \\
\text { Barreiras }\end{array}$ & $\begin{array}{l}\text { Floresta } \\
\text { tropical } \\
\text { subperenifólia }\end{array}$ & $\begin{array}{l}\text { Linhares } \\
\quad(E S)\end{array}$ & $\begin{array}{l}\text { Culturas anuais (feijão e milho) } \\
\text { por } 11 \text { anos. Nos } 3 \text { anos } \\
\text { precedentes à amostragem, o } \\
\text { solo permaneceu coberto com } \\
\text { capim-colonião (P. maximum). }\end{array}$ & $\begin{array}{l}\text { Adubação } \\
\text { fosfatada anual } \\
\text { e calagem } \\
\text { ocasional }\end{array}$ \\
\hline $\begin{array}{l}\text { Latossolo Amarelo } \\
\text { distrófico (LAd) }\end{array}$ & $\begin{array}{l}\text { Produto de } \\
\text { alteração de } \\
\text { gnaisse } \\
\text { granítico }\end{array}$ & $\begin{array}{l}\text { Floresta } \\
\text { tropical } \\
\text { subcaducifólia }\end{array}$ & $\begin{array}{l}\text { Lavras } \\
\text { (MG) }\end{array}$ & $\begin{array}{l}\text { Culturas anuais por mais } \\
\text { de } 10 \text { anos. }\end{array}$ & $\begin{array}{l}\text { Adubação } \\
\text { fosfatada anual } \\
\text { e calagem } \\
\text { ocasional }\end{array}$ \\
\hline $\begin{array}{l}\text { Latossolo Vermelho } \\
\text { ácrico (LVw) }\end{array}$ & $\begin{array}{l}\text { Sedimentos } \\
\text { da cobertura } \\
\text { detrítica } \\
\text { terciária }\end{array}$ & $\begin{array}{l}\text { Cerrado } \\
\text { tropical } \\
\text { subcaducifólio }\end{array}$ & $\begin{array}{l}\text { Uberlândia } \\
\qquad(\mathrm{MG})\end{array}$ & $\begin{array}{l}\text { Culturas anuais por mais } \\
\text { de } 10 \text { anos. }\end{array}$ & $\begin{array}{l}\text { Adubação } \\
\text { fosfatada pesada } \\
\text { e calagem anual }\end{array}$ \\
\hline $\begin{array}{l}\text { Latossolo Vermelho } \\
\text { distroférrico (LVdfg) }\end{array}$ & $\begin{array}{l}\text { Produto da } \\
\text { alteração de } \\
\text { gabro }\end{array}$ & $\begin{array}{l}\text { Floresta } \\
\text { tropical } \\
\text { subperenifólia }\end{array}$ & $\begin{array}{l}\text { Lavras } \\
\text { (MG) }\end{array}$ & $\begin{array}{l}\text { Culturas anuais por mais de } 10 \\
\text { anos. N os } 5 \text { anos precedentes à } \\
\text { amostragem, o solo permaneceu } \\
\text { coberto com braquiária } \\
\text { (B. decumbens). }\end{array}$ & $\begin{array}{l}\text { Adubação } \\
\text { fosfatada anual } \\
\text { e calagem } \\
\text { ocasional }\end{array}$ \\
\hline $\begin{array}{l}\text { Latossolo Vermelho } \\
\text { distroférrico (LVdft) }\end{array}$ & $\begin{array}{l}\text { Produto da } \\
\text { alteração de } \\
\text { tufito }\end{array}$ & $\begin{array}{l}\text { Floresta } \\
\text { tropical } \\
\text { subcaducifólia }\end{array}$ & $\begin{array}{l}\text { Patos de Minas } \\
\qquad(\mathrm{MG})\end{array}$ & $\begin{array}{l}\text { Culturas anuais por mais de } \\
15 \text { anos. Nos } 5 \text { anos precedentes } \\
\text { à amostragem, o solo } \\
\text { permaneceu coberto com } \\
\text { braquiária. }\end{array}$ & $\begin{array}{l}\text { Adubação } \\
\text { fosfatada anual } \\
\text { e calagem } \\
\text { ocasional }\end{array}$ \\
\hline
\end{tabular}

Quadro 2. Atributos químicos e físicos dos $\operatorname{solos}^{(1)}$

\begin{tabular}{|c|c|c|c|c|c|c|c|c|c|c|}
\hline \multirow{2}{*}{ Atributo } & \multicolumn{2}{|c|}{ LAx } & \multicolumn{2}{|c|}{ LAd } & \multicolumn{2}{|c|}{ LVw } & \multicolumn{2}{|c|}{ LVdfg } & \multicolumn{2}{|c|}{ LVdft } \\
\hline & NC & $\mathrm{C}$ & NC & $\mathrm{C}$ & NC & $\mathrm{C}$ & NC & $\mathrm{C}$ & NC & $\mathrm{C}$ \\
\hline pH em água & 5,7 & 5,9 & 5,4 & 5,7 & 4,9 & 5,8 & 5,5 & 6,1 & 4,9 & 5,2 \\
\hline $\mathrm{Al}^{3+}\left(\mathrm{cmol}_{\mathrm{c}} \mathrm{dm}^{-3}\right)$ & 0,0 & 0,0 & 0,6 & 0,0 & 0,9 & 0,0 & 0,0 & 0,0 & 0,8 & 0,2 \\
\hline $\mathrm{Ca}^{2+}\left(\mathrm{cmol}_{\mathrm{c}} \mathrm{dm}^{-3}\right)$ & 3,2 & 3,0 & 1,7 & 3,4 & 1,2 & 3,7 & 3,2 & 7,2 & 2,3 & 2,2 \\
\hline $\mathrm{Mg}^{2+}\left(\mathrm{cmol}_{\mathrm{c}} \mathrm{dm}^{-3}\right)$ & 0,8 & 1,2 & 0,8 & 0,9 & 0,1 & 0,2 & 0,2 & 0,3 & 0,9 & 1,1 \\
\hline $\mathrm{K}+\left(\mathrm{mg} \mathrm{dm}^{-3}\right)$ & 90 & 73 & 101 & 172 & 36 & 59 & 153 & 162 & 64 & 166 \\
\hline $\mathrm{H}+\mathrm{Al}\left(\mathrm{cmol}_{\mathrm{c}} \mathrm{dm}^{-3}\right)$ & 4,0 & 2,3 & 6,3 & 3,6 & 9,8 & 2,9 & 4,0 & 2,3 & 12,3 & 7,0 \\
\hline P-M ehlich-1 (mg dm$\left.{ }^{-3}\right)$ & 4 & 22 & 4 & 12 & 2 & 45 & 3 & 7 & 78 & 63 \\
\hline P-Resina (mg dm-3) & 11 & 23 & 10 & 27 & 7 & 48 & 17 & 18 & 91 & 57 \\
\hline$B\left(\mathrm{mg} \mathrm{dm}^{-3}\right)$ & 0,31 & 0,31 & 0,15 & 0,23 & 0,23 & 0,26 & 0,26 & 0,29 & 0,26 & 0,26 \\
\hline $\mathrm{Cu}\left(\mathrm{mg} \mathrm{dm}^{-3}\right)$ & 0,9 & 3,0 & 0,5 & 2,0 & 1,6 & 1,0 & 4,1 & 5,1 & 7,4 & 10,2 \\
\hline $\mathrm{Fe}\left(\mathrm{mg} \mathrm{dm}^{-3}\right)$ & 176 & 150 & 150 & 159 & 329 & 100 & 109 & 116 & 526 & 177 \\
\hline $\mathrm{Mn}\left(\mathrm{mg} \mathrm{dm}^{-3}\right)$ & 66 & 57 & 27 & 75 & 19 & 6 & 94 & 21 & 114 & 78 \\
\hline $\mathrm{Zn}\left(\mathrm{mg} \mathrm{dm}^{-3}\right)$ & 2,5 & 5,7 & 1,3 & 7,9 & 1,4 & 2,8 & 2,7 & 8,3 & 2,6 & 4,7 \\
\hline $\mathrm{S}\left(\mathrm{cmol}_{\mathrm{c}} \mathrm{dm}^{-3}\right)$ & 4,2 & 4,4 & 2,8 & 4,7 & 1,4 & 4,1 & 3,8 & 7,9 & 3,4 & 3,7 \\
\hline $\mathrm{t}\left(\mathrm{cmol}_{\mathrm{c}} \mathrm{dm}^{-3}\right)$ & 4,2 & 4,4 & 2,9 & 4,7 & 2,3 & 4,1 & 3,8 & 7,9 & 4,2 & 3,9 \\
\hline $\mathrm{T}\left(\mathrm{cmol}_{\mathrm{c}} \mathrm{dm}^{-3}\right)$ & 8,2 & 6,7 & 9,1 & 8,3 & 11,2 & 7,0 & 7,8 & 10,2 & 15,7 & 10,7 \\
\hline V (\%) & 51 & 66 & 31 & 57 & 13 & 58 & 49 & 77 & 21 & 35 \\
\hline m (\%) & 0 & 0 & 18 & 0 & 39 & 0 & 0 & 0 & 19 & 5 \\
\hline$C$ org. $\left(\mathrm{g} \mathrm{kg}^{-1}\right)$ & 22,3 & 13,6 & 21,9 & 19,5 & 26,4 & 19,1 & 30,0 & 23,6 & 49,5 & 31,9 \\
\hline Argila $\left(\mathrm{g} \mathrm{kg}^{-1}\right)$ & 440 & 430 & 360 & 380 & 670 & 630 & 640 & 660 & 580 & 600 \\
\hline Silte $\left(\mathrm{g} \mathrm{kg}^{-1}\right)$ & 90 & 110 & 200 & 180 & 120 & 100 & 160 & 190 & 240 & 230 \\
\hline Areia $\left(\mathrm{g} \mathrm{kg}^{-1}\right)$ & 470 & 460 & 440 & 440 & 210 & 270 & 200 & 150 & 180 & 170 \\
\hline
\end{tabular}

(1) LAx: Latossolo Amarelo coeso; LAd: Latossolo Amarelo distrófico; LVw: Latossolo Vermelho ácrico; LVdfg: Latossolo Vermelho distroférrico desenvolvido de gabro; LVdft: Latossolo Vermel ho distroférrico desenvolvido de tufito; NC: não cultivado; C: cultivado. 
diferencial (ATD) no resíduo do tratamento com ditionito-citrato-bicarbonato (DCB) (Mehra \& J ackson, 1960), após secagem em estufa e destorroamento suave em gral de ágata.

As formas de $\mathrm{P}$ foram determinadas conforme Chang \& J ackson (1957) e Hedley et al. (1982). O método de Chang \& J ackson (1957) permite separar inicialmente o $\mathrm{P}$ solúvel, usando $\mathrm{NH}_{4} \mathrm{Cl}$, e, em seguida, os compostos de P-Al, com $\mathrm{NH}_{4} \mathrm{~F}$ a pH 7,0. $A$ amostra é então submetida à extração das formas $\mathrm{P}-\mathrm{Fe}$, com $\mathrm{NaOH}$, e do $\mathrm{P}-\mathrm{Ca}$, com $\mathrm{H}_{2} \mathrm{SO}_{4}$. A etapa final consiste na determinação do $\mathrm{P}$-Fe presente na forma de compostos insolúveis, através da solução, por redução com $\mathrm{Na}_{2} \mathrm{~S}_{2} \mathrm{O}_{4}$ na presença de citrato. $\mathrm{O}$ método proposto por Hedley et al. (1982) eadaptado por Pavan \& Chaves (1996) permite quantificar as formas lábeis de $\mathrm{P}$, que incluem $\mathrm{P}$ disponível ( $\mathrm{P}$ resina), $\mathrm{P}$ adsorvido à superfície dos colóides $\left[(\mathrm{Pi}+\mathrm{Po})-\mathrm{NaHCO}_{3}\right]$ e o $\mathrm{P}$ microbiano $[(\mathrm{Pi}+\mathrm{Po})$ $\mathrm{NaHCO}_{3} / \mathrm{CH}_{3} \mathrm{Cl}$ ]; formas pouco lábeis, que incluem o P inorgânico ligado a Fe eAl e o P orgânico ligado a compostos húmicos [( $\mathrm{Pi}+\mathrm{Po})-\mathrm{NaOH}]$; as formas não-lábeis, que incluem o $\mathrm{P}$ ligado ao $\mathrm{Ca}(\mathrm{P}-\mathrm{HCl})$, considerado relativamente insolúvel, e as formas mais estáveis de $\mathrm{Po}$ e $\mathrm{Pi}$ (P-residual), determinadas após digestão com $\mathrm{H}_{2} \mathrm{SO}_{4} \mathrm{eH}_{2} \mathrm{O}_{2}$.

$\mathrm{N}$ os extratos do bicarbonato: bicarbonato após tratamento com clorofórmio, extrato acidificado do bicar bonato, hidróxido de sódio e hidróxido de sódio com sonificação, procedeu-se à determinação do $P$ com e sem digestão, para a separação do Pi e do Po. Para a extração com resina, utilizaram-se sacos de pol ietileno de $400 \mathrm{~mm}$ demalha, que continham 0,6 g de resina de troca aniônica DOWAX2-X18 saturada com bicarbonato. Em todos os extratos, o P foi determinado por colorimetria, segundo Murphy \& Riley (1962), sendo procedidas três repetições.

\section{Análises estatísticas}

Foram realizados testes de média (Tukey) e correlações lineares (Pearson), empregando-se o Sistema deAnálises Estatísticas e Genéticas (SAEG) da Central de Processamentos de Dados da UFV.

\section{RESULTADOS E DISCUSSÃO}

Observou-se que todos os solos não cultivados apresentaram val ores depH entre 5,4 e6,1, à exceção doLVw eLVdft, com valores ligei ramente mais baixos (Quadro 2). Os solos cultivados (C) apresentaram valores nulos ou quase nulos de Al trocável, enquanto, entre os não cultivados (NC), o LVw eLVdft apresentaram valores mais elevados, em torno de $0,8 \mathrm{cmol}_{\mathrm{C}} \mathrm{dm}^{-3}$. Em termos de macronutrientes, todos os solos, exceto o LVw e LVdfg, apresentaram teores médios de Ca e Mg e médios ealtos de K. Os val ores de $\mathrm{P}$ extraído por Mehlich-1 foram altamente discrepantes entre solos não cultivados ( 2 a $78 \mathrm{mg} \mathrm{dm}^{-3}$ ) e cultivados ( 7 a $63 \mathrm{mg} \mathrm{dm}^{-3}$ ). No caso do LVdft, os valores foram bem mais elevados até mesmo no solo não cultivado, indicando herança do seu material de origem, em consonância com o teor $\mathrm{deP}_{2} \mathrm{O}_{5}$ proveniente do ataque sulfúrico (Quadro 3), índice confiável do $\mathrm{P}$ total, segundo Lemos et al. (1997). Os resultados deP-Resina mostraram valores mais el evados que o P-M ehlich-1 em todos os casos, à exceção do LVdft-C.

Quanto aos micronutrientes, nos solos não cultivados, à exceção do $B$, os maiores valores foram registrados nos LVdf, em consonância com a maior riqueza das rochas máficas que originaram estes solos (Curi \& Franzmeier, 1987; Resende et al., 1999a).

Em todos os extratos, o teor de Fe decresceu na ordem $L V d f t>L V d f g>L V w>L A d>L A x$. A relação $\mathrm{Fe}_{\mathrm{d}} / \mathrm{Fe}_{\mathrm{d}}$ é utilizada como um índice de cristal inidade dos óxidos de Fe no solo, estando os valores mais el evados relacionados com a presença de inibidores decristal ização, que podem dificul tar a transformação da ferrihidrita em óxidos mais bem cristalizados. Assim, a ocorrência devalores mais bai xos (Quadro 4) está condizente com a natureza latossól ica dos sol os estudados (Kämpf et al., 1988; Kämpf \& Curi, 2000).

Os valores de $\mathrm{Fe}_{2} \mathrm{O}_{3}$ obtidos pel o ataque sulfúrico variaram de 11 a $379 \mathrm{~g} \mathrm{~kg}^{-1}$, situando-se dentro dos limites requeridos para as respectivas classes de solos, enquanto os índices $\mathrm{Ki}$ e $\mathrm{Kr}$ (Quadro 3) diminuíram do LAx para o LVdft, indicando, neste sentido, o aumento do caráter oxídico. Este fato foi corroborado pel os resultados obtidos com a DRX da argila desferrificada, que indicou a existência de diferentes proporções de caulinita e gibbsita (Figura 1). Da mesma forma, os valores de Ct/ (Ct + Gb) (Quadro 4), obtidos a partir dos termogramas da fração argila desferrificada, apresentaram clara

\section{Quadro 3. Óxidos do ataque sulfúrico e índices Ki e $\mathrm{Kr}$}

\begin{tabular}{|c|c|c|c|c|c|c|c|}
\hline Solo & $\mathrm{SiO}_{2}$ & $\mathrm{Al}_{2} \mathrm{O}_{3}$ & $\mathrm{Fe}_{2} \mathrm{O}_{3}$ & $\mathrm{TiO}_{2}$ & $\mathbf{P}_{2} \mathbf{O}_{5}$ & $\mathbf{K i}$ & $\mathbf{K r}$ \\
\hline & \multicolumn{5}{|c|}{$\mathrm{g} \mathrm{kg}^{-1}$} & & \\
\hline LAx-NC & 176 & 172 & 11 & 12 & 0,07 & 1,74 & 1,67 \\
\hline LAx-C & 178 & 182 & & 12 & 0,07 & 1,66 & 1,55 \\
\hline LAd-NC & 90 & 141 & 31 & 8 & 0,12 & 1,09 & 0,96 \\
\hline LAd-C & 103 & 174 & 51 & 11 & 0,23 & 1,00 & 0,85 \\
\hline LVw-NC & 129 & 268 & 106 & 20 & 0,34 & 0,82 & 0,65 \\
\hline LVw-C & 189 & 323 & 123 & 23 & 0,46 & 1,00 & 0,80 \\
\hline LVdfg-NC & 158 & 279 & 220 & 27 & 0,49 & 0,96 & 0,64 \\
\hline LVdfg-C & 154 & 271 & 177 & 31 & 0,68 & 0,96 & 0,68 \\
\hline LVdft-NC & 53 & 239 & 326 & 74 & 8,30 & 0,38 & 0,20 \\
\hline LVdft-C & 41 & 226 & 379 & 89 & 10,37 & 0,31 & 0,15 \\
\hline
\end{tabular}

NC: não cultivado; C: cultivado. 
Quadro 4. Cor e alguns atributos químicos e mineralógicos dos Latossolos estudados sob diferentes condições de cultivo

\begin{tabular}{|c|c|c|c|c|c|c|c|c|c|}
\hline Solo & Cor (úmida) & $\mathbf{F e}_{0}^{(1)}$ & $\mathbf{F} \mathbf{e}_{\mathrm{d}}^{(2)}$ & $\mathbf{C t}^{(3)}$ & $\mathbf{G} \mathbf{b}^{(4)}$ & $\mathbf{F e}_{\mathrm{o}} / \mathbf{F e}_{\mathrm{d}}$ & $\mathbf{G t} / \mathbf{G t}+\mathbf{H m}^{(5)}$ & $\mathbf{G t} / \mathbf{C t}+\mathbf{G b}$ & CMAF $^{(6)}$ \\
\hline & & \multicolumn{4}{|c|}{$\mathrm{g} \mathrm{kg}^{-1}$} & & & & $\mathrm{mg} \mathrm{kg}^{-1}$ \\
\hline LAx-NC & 10YR 4/2 & 0,3 & 1 & 260 & 10 & 0,30 & 1,00 & 0,96 & 476 \\
\hline LAx-C & 10YR 4/1 & 0,7 & 11 & 230 & 10 & 0,06 & 1,00 & 0,96 & 324 \\
\hline LAd-NC & $7,5 Y R$ 3/4 & 1,9 & 34 & 110 & 60 & 0,06 & 1,00 & 0,65 & 621 \\
\hline LAd-C & $7,5 Y R$ R/3 & 1,8 & 46 & 100 & 90 & 0,04 & 1,00 & 0,53 & 676 \\
\hline LVw-NC & $2,5 Y R \quad 3 / 4$ & 2,4 & 55 & 230 & 270 & 0,04 & 0,17 & 0,46 & 1.233 \\
\hline LVw-C & $2,5 Y R$ 3/4 & 2,1 & 61 & 190 & 110 & 0,03 & 0,14 & 0,63 & 1.052 \\
\hline LVdfg-NC & $2,5 Y R 2,5 / 4$ & 2,9 & 87 & 240 & 200 & 0,03 & 0,34 & 0,55 & 1.393 \\
\hline LVdfg-C & 2,5YR 3/4 & 2,8 & 85 & 230 & 220 & 0,03 & 0,45 & 0,51 & 1.393 \\
\hline LVdft-NC & $5 Y R 3 / 3$ & 5,3 & 121 & 0 & 260 & 0,04 & 0,09 & 0,00 & 3.000 \\
\hline LVdft-C & $5 Y R 3 / 3$ & 5,1 & 117 & 0 & 300 & 0,04 & 0,12 & 0,00 & 2.092 \\
\hline
\end{tabular}

${ }^{(1)}$ Ferro extraído com oxalato de amônio. ${ }^{(2)}$ Ferro extraído com ditionito-citrato-bicarbonato. ${ }^{(3)}$ Caulinita. ${ }^{(4)}$ Gibbsita. ${ }^{(5)}$ Gt: goethita e Hm: hematita. ${ }^{(6)}$ Capacidade máxima de adsorção de fósforo. NC: não cultivado; C: cultivado.

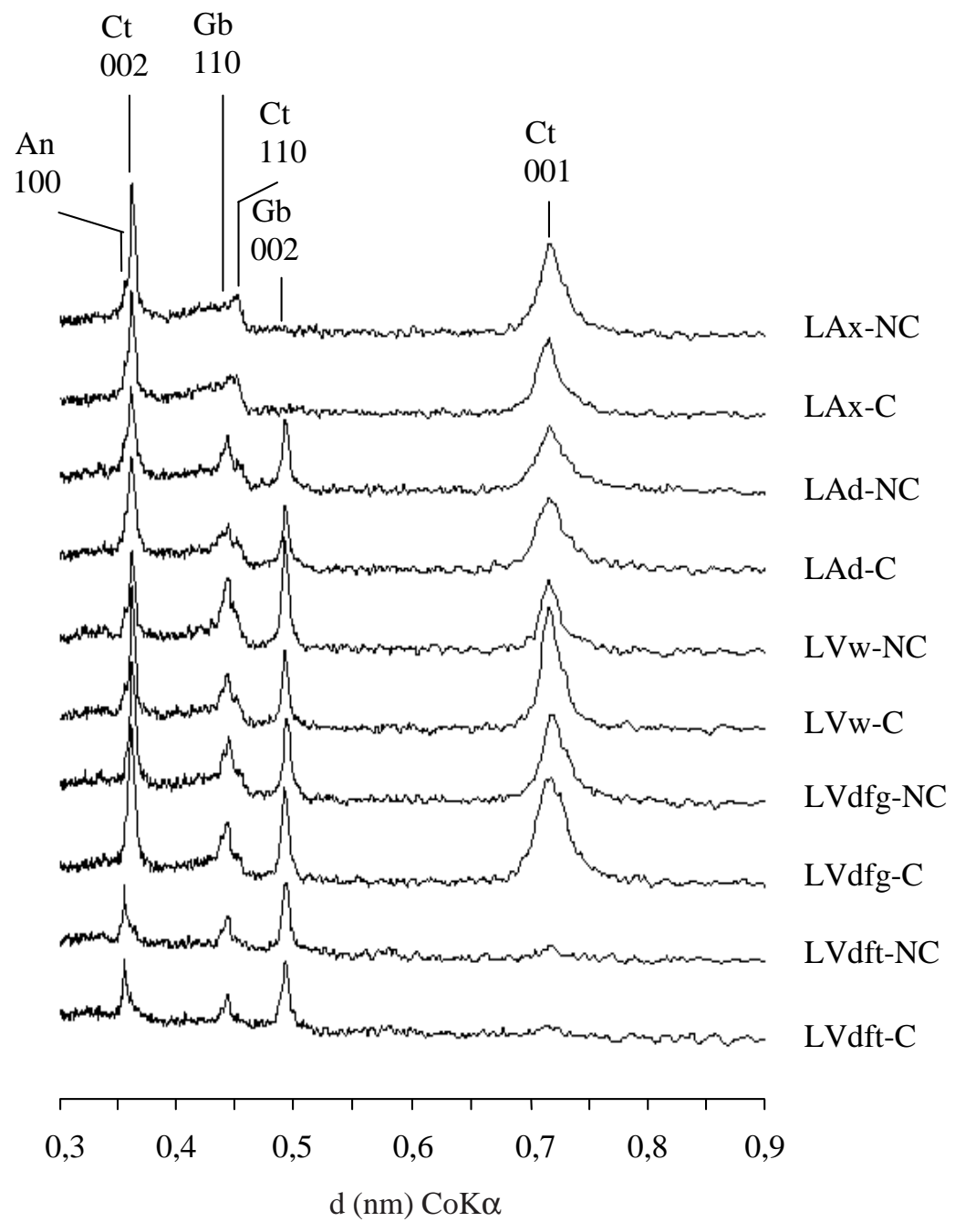

Figura 1. Difratogramas de raios-X da fração argila desferrificada. An: Anatásio; Ct: Caulinita; Gb: Gibbsita. 
tendência de decréscimo do LAx (caulinítico) para o LVdft (gibbsítico).

Os valores da capacidade máxima de adsorção de $P$, fornecidos pelo parâmetro $b$ da equação de Langmuir, variaram de 324 a $3.000 \mathrm{mg} \mathrm{kg}^{-1}$ (Quadro 4), havendo uma tendência de os solos cultivados apresentarem val ores mais baixos do que seus equivalentes não cultivados. As prováveis explicações para estefato são: (a) a calagem provocou uma diminuição das cargas positivas, diminuindo a adsorção de $\mathrm{P}$; (b) a calagem promoveu maior decomposição de matéria orgânica (Quadro 2), podendo ter gerado frações de menor peso mol ecular e, ou, de maior solubilidade (a qual é incrementada com a el evação do pH), competindo, desta maneira, com o $\mathrm{P}$ pel os sítios de troca (Silva et al., 2000), e (c) a adubação fosfatada ao longo dos anos pode ter promovido a remoção de parte dos elementos em sol ução (Ca, FeeAl) por precipitação com P (Lindsay, 1979).
Constatou-se correlação negativa, significativa, entre a razão $\mathrm{Ct} /(\mathrm{Ct}+\mathrm{Gb})$ e a CMAF $(r=-0,91)$, em concordância com Ker (1995), o que demonstra a maior efetividade da gibbsita em relação à caulinita na adsor ção de $\mathrm{P}$, embora haja um aumento no teor de $\mathrm{Fe}_{\mathrm{d}}$ neste mesmo sentido.

A análise dos difratogramas de raios- $X$ da fração argila ferro-concentrada (Figura 2 ) indicou a presença de goethita (reflexo a 4,18 nm) em todos os solos, enquanto, no LVw, LVdfg e LVdft, a presença de hematita foi evidenciada pelos reflexos a 0,368; 0,$269 ; 0,253$ e 0,220 nm. Os val ores de Gt/(Gt + Hm) diminuíram gradualmente do LAx para o LVdft ( $\mathrm{LAx}=\mathrm{LAd}<\mathrm{LVdfg}<\mathrm{LVw}<\mathrm{LVdft}$ ), indicando a ocorrência isolada de goethita no LAx e LAd, um predomínio de hematita no LVdft e proporções intermediárias no LVdfg e LVw (Quadro 4).

A presença de hematita no LVw, LVdfg e LVdft está em acordo com a coloração das amostras

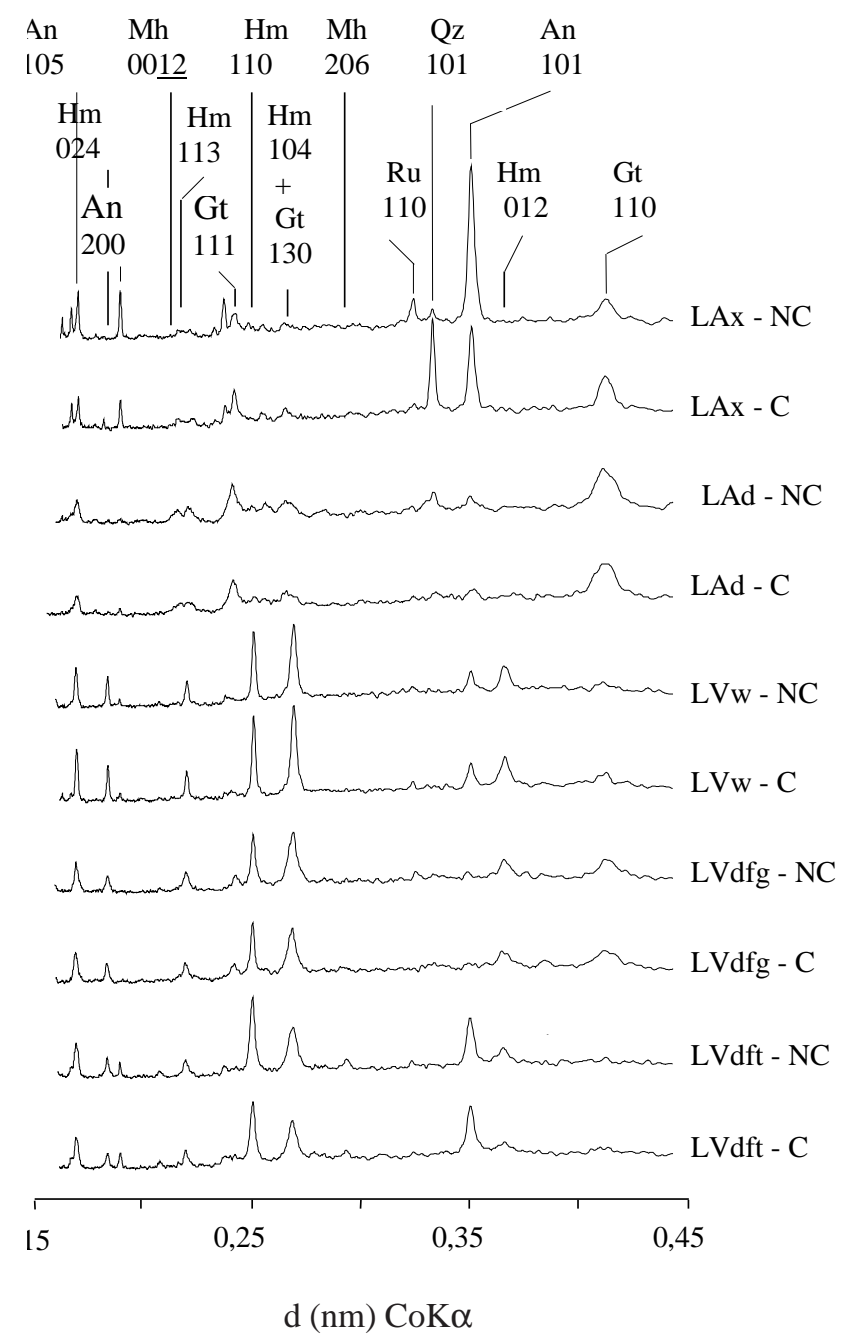

Figura 2. Difratogramas de raios-X da fração argila ferro-concentrada. Gt = Goethita; $\mathbf{H m}=$ Hematita; An =Anatásio; Qz = Quartzo; Ru = Rutilo; Mh = Maghemita. 
(Quadro 4), uma vez que a presença deste mineral na fração argila, mesmo em pequenas quantidades, é suficiente para tornar a amostra avermelhada, mascarando a cor amarelada da goethita (Scheffer et al., 1958; Resende, 1976). A razão $\mathrm{Gt} /(\mathrm{Gt}+\mathrm{Hm})$ apresentou correlação negativa significativa com a CMAF $(r=-0,80)$, indicando que, para valores mais elevados dessa razão (amostra mais goethítica), correspondem val ores mais baixos de CMAF, eviceversa. O que pode explicar esta aparente incongruência é o fato de que, dentro do grupo de sol os estudados, no mesmo senti do em que aumenta o caráter goethítico, do LVdft para o LAx, há redução acentuada no teor de $\mathrm{Fe}_{d}$, que apresentou al to índice de correlação com a CMAF $(r=0,92)$. O efeito da goethita na adsorção de $P$, portanto, foi superado pelo efeito da soma total de óxidos (goethita, hematita e gibbsita), que foi mais baixa no LAx e aumentou no sentido do LVdft.

Comparando pares de solos cultivados e não cultivados, verificou-se que os resultados de $\mathrm{P}_{2} \mathrm{O}_{5}$ "total" (Quadro 3) indicaram que, à exceção do LAx, os solos receberam muito $\mathrm{P}$ durante o período de cultivo. Como exemplo, pode ser citado o incremento verificado no LVw-C em comparação ao LVw-NC $\left(0,12 \mathrm{~g} \mathrm{~kg}^{-1}\right)$. Admitindo uma densidade de $1,3 \mathrm{t} \mathrm{m}^{-3}$, esteval or representou, nos primeiros $0,2 \mathrm{~m}$ (camada arável), um acréscimo de $312 \mathrm{~kg} \mathrm{ha}^{-1}$ de $\mathrm{P}_{2} \mathrm{O}_{5}$.

Os valores de $\mathrm{Ki}$ e Kr (Quadro 3), que retratamo grau de intemperização do solo, correlacionaram-se de maneira negativa com a CMAF $(r=-0,86$ para os dois índices), em consonância com os resultados de Curi et al. (1988).

Apesar da diversidade do material de origem dos solos, a substituição de Fe por Al na estrutura da goethita, que afeta a área superficial específica deste mineral, permaneceu dentro do intervalo de 0,18 a $0,33 \mathrm{~mol} \mathrm{~mol}^{-1}$. Por não ter sido constatada uma tendência definida e pelo baixo efeito relativo da goethita na adsorção de $\mathrm{P}$ destes solos, conforme discutido anteriormente, é de se esperar que a influência da substituição de Fe por Al na adsorção de P não seja apreciável.

Não houve correlação significativa entre teor de argila eCMAF ( $r=0,58)$ (Motta, 1999), evidenciando a preponderância da influência das características químicas e mineralógicas na adsorção de $\mathrm{P}$ dos Latossolos estudados.

O balanço entre a riqueza da rocha de origem eo grau de desenvolvimento pedogenético, que determina o grau de empobrecimento do solo, combinados com o efeito do manejo, ocasionaram diferenças na magnitude das formas de $P$ encontradas nestes solos. $\mathrm{Na}$ figura 3 , está comparada a magnitude das formas de $\mathrm{P}$, conforme Heddley et al. (1982), entre solos não cultivados e cultivados, em termos de percentual do P total, evidenciando que: (a) em todas as situações, predomina Pt-NaOH; (b) nos solos não cultivados,
Po-NaOH éa segunda forma mais importante, e (c) nos solos cultivados, $\mathrm{Pi}-\mathrm{NaOH}$ é a segunda forma mais importante. Em outras palavras, predominam formas pouco lábeis, as quais, com o cultivo, tendem a passar das formas orgânicas associadas a compostos húmicos (NC) para as formas inorgânicas ligadas a FeeAl (C).

Com exceção do LVdft, os solos cultivados apresentaram P disponível, estimado pelo P-Resina (Figura 3) mais elevado, graças à adubação fosfatada durante o cultivo. Dada a presença de pastagem há mais de cinco anos, é possível que tenha ocorrido mai or extração de $\mathrm{P}$ pela B. decumbens, explicando o fato de o LVdft cultivado apresentar teor de P disponível mais baixo que o não cultivado.

De modo geral, os teores de P-Resina obtidos pelo fracionamento (Figura 3) foram mais baixos do que os obtidos pela análise de rotina (Quadro 2). I sso pode ser atribuído ao uso da fração TFSA eà maior relação sol o:extrator neste método, ao passo que, no fracionamento, foi utilizado sol o passado em peneira de $0,15 \mathrm{~mm}$, após moagem, procedimento esse que pode expor novos sítios de adsorção, antes inacessíveis, reduzindo o P na solução de equilíbrio. A exceção observada para o LVdft, onde o teor de P. Resina obtido pel o fracionamento foi muito superior ao da análise de rotina, deveu-se possivelmente à grande quantidade de $\mathrm{P}$ presente no interior dos agregados (Moura Filho \& Buol, 1976; Linquist et al., 1997), que éliberada com a moagem e compensa a exposição de novos sítios de adsorção.

Conforme observações de campo e durante o preparo das amostras para análise, ficou nítido que os agregados do LVdft foram os mais resistentes dentro do grupo de sol os estudados. O P-Microbiano não diferiu muito entre solos cultivados e não cultivados, exceto para o LVdft, atingindo o dobro no solo não cultivado, em razão da sua mai or riqueza em matéria orgânica (Quadro 2).

O P-Bicarbonato, especialmente a fração inorgânica ( $\mathrm{Pi})$, foi mais el evado nos sol os cultivados, o que indica a contribuição da adubação fosfatada durante o período de cultivo. Com exceção do LVdft e do LVw-C, a fração orgânica do P-Bicarbonato foi maior do que a inorgânica, constituindo 75 a $90 \%$ nos sol os não cultivados e 52 a $58 \%$ nos cultivados. No LVdft, o Pi-Bicarbonato foi superior ao Po tanto em sol os cultivados quanto não cultivados. Também o P-NaOH, especialmente a fração inorgânica, foi maior nos sol os cultivados, o que reflete também o efeito da adubação fosfatada. Exceto para o LVdft, a fração orgânica (52 a 66 \% do Pt-NaOH) foi maior que a inorgânica nos sol os não cultivados, ocorrendo o inverso nos solos cultivados, onde a fração inorgânica constituiu 59a 69 \% do Pt-NaOH. NoLVdft, a fração inorgânica foi maior, independentemente do histórico de uso. O P-NaOH, fração equival enteaoP menos prontamente disponível, ligado mais fortemente a FeeAl (Hedley et al., 1982; Tiessen et 
NÃO CULTIVADO

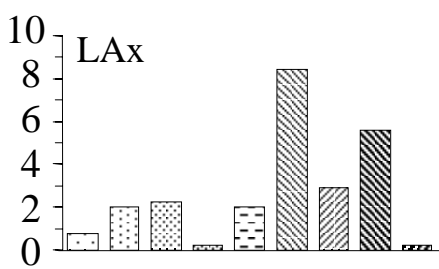

15

12

9

6

3

0

12

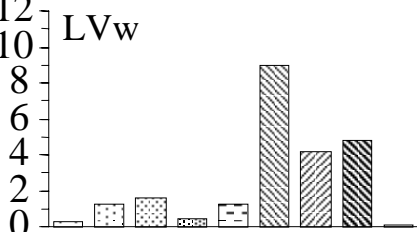

15

12

9

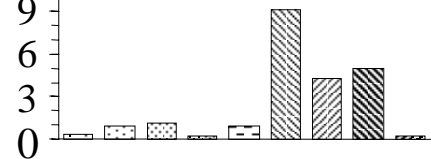

30 LVdft

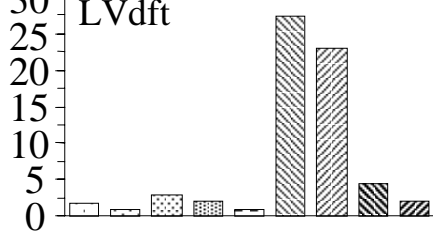

CULTIVADO

LAx

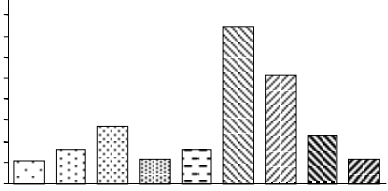

LAd

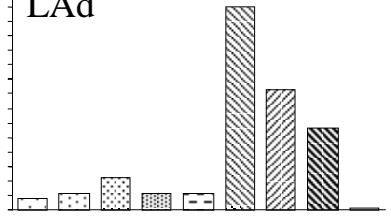

LVw

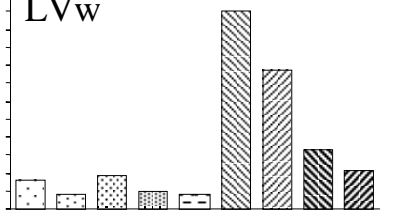

LVdfg

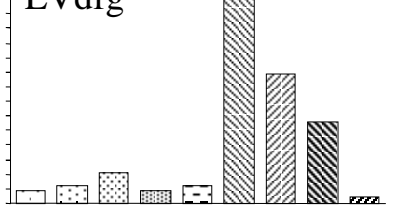

LVdft

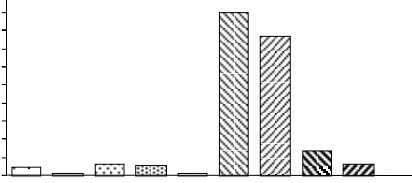

FORMAS DE P

$\square$ P-resina $\because$ P-microbiano $\square$ Pt-bicarbonato

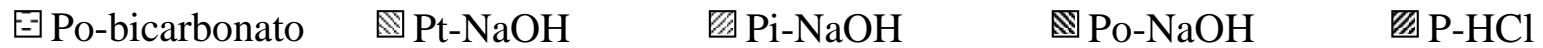

Figura 3. Distribuição percentual das formas de $P$, conforme Heddley et al. (1982), em relação ao $P$ total, nos solos estudados.

al., 1983), aumentou gradativamente do LAx para o LVdft (Figura 3), na mesma ordem da CMAF (Quadro 4). J á o P-HCl, que correspondeao P ligado a $\mathrm{Ca}$, foi maior nos solos cultivados, à exceção dos LVdft, o que pode ser atribuído ao maior conteúdo deCa introduzido pel as cal agens ao longo do período de cultivo.

Deacordo com a figura 3, a maior parte do P total do sol o está na fração residual, sendo os teores deP ligados a FeeAl (Pi-NaOH) bastante baixos. Foram obtidas correlações positivas, significativas, entre CMAF eP-Resina $(r=0,89)$, P Microbiano $(r=0,83)$ eP-Residual $(r=0,78)$.
Os valores de P-residual encontrados neste trabalho ficaram bem acima dos val ores registrados por Goedert (1987) e Marques (2000). U ma possível explicação seria ofato deque dois dos cinco L atossol os estudados são derivados de rochas máficas. Além disso, esta fração pode estar induindo al gum $\mathrm{P}$ ligado a Fe eAl que não foi extraído pelo $\mathrm{NaOH}$.

As formas P-Al e P-Fe, determinadas segundo Chang \& J ackson (1957), dominaram amplamente sobre o P-Ca (Quadro 5). Os aumentos relativos de P-Al e P-Fecom o cultivo indicam a importância das adubações fosfatadas sobre estas frações. O P-AI predominou no LAx, ondea razão P-Fe/ (P-Al + P-Fe) 
Quadro 5. Fósforo ligado a Al, Fe e Ca, segundo Chang \& J ackson (1957), em cinco Latossolos previamente cultivados (C) ou não cultivados (NC)

\begin{tabular}{|c|c|c|c|c|c|c|c|c|c|c|}
\hline \multirow{2}{*}{ Forma de fósforo } & \multicolumn{2}{|c|}{ LAx } & \multicolumn{2}{|c|}{ LAx } & \multicolumn{2}{|c|}{ LAd } & \multicolumn{2}{|c|}{ LVw } & \multicolumn{2}{|c|}{ LVdft } \\
\hline & NC & C & NC & C & NC & C & NC & C & NC & C \\
\hline & & & & 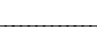 & $-\mathrm{mg}$ & 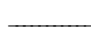 & 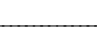 & 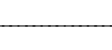 & 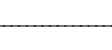 & - \\
\hline $\mathrm{P}-\mathrm{Al}$ & 22 & 50 & 20 & 63 & 23 & 79 & 35 & 87 & 703 & 504 \\
\hline $\mathrm{P}-\mathrm{Fe}$ & 10 & 39 & 28 & 91 & 35 & 85 & 66 & 110 & 839 & 839 \\
\hline $\mathrm{P}-\mathrm{Ca}$ & 1 & 2 & 2 & 3 & 5 & 33 & 6 & 7 & 283 & 341 \\
\hline $\mathrm{P}-\mathrm{Ca} /(\mathrm{P}-\mathrm{Al}+\mathrm{P}-\mathrm{Fe})$ & 0,03 & 0,02 & 0,04 & 0,02 & 0,06 & 0,20 & 0,06 & 0,04 & 0,18 & 0,25 \\
\hline
\end{tabular}

foi 0,31 no solo não cultivado e 0,44 no cultivado, enquanto o P-Fe predominou no restante dos solos, onde os valores daquela razão variaram de 0,52 a 0,65 , em consonância com os baixos teores de $\mathrm{Fe}$ "total" no LAx (Quadro 3).

Chama a atenção o fato de ser o val or de P-Ca no LAx cultivado muito menor que o seu equivalente pelo método de Hedley et al. (1982), $\mathrm{P}-\mathrm{HCl}$. Os maiores val ores da relação P-Ca / (P-Al + P-Fe) foram observados no LVw-C e nos LVdft (NC e C). No primeiro caso, isto se deveu ao uso de calagens e adubações fosfatadas pesadas ao longo dos anos, sendo raro, no Brasil, encontrar-se LVw com teores tão el evados de P disponível (Quadro 2). No caso do LVdft, a riqueza original do solo em $P$ ajuda a explicar tais resultados, pois não há muita variação nas concentrações entre o solo cultivado e não cultivado.

\section{CONCLUSÕES}

1. A adsor ção, oteor total eas formas deP ligadas mais fortementea Al eFeaumentaram com o caráter oxídico dos solos.

2. O cultivo influenciou, de modo diferenciado, a adsorção de $\mathrm{P}$ e aumentou em todos os solos as formas de $\mathrm{P}$ ligado a $\mathrm{Ca}$.

3. As formas pouco lábeis de P predominaram nos Latossolos estudados, destacando-se as orgânicas associadas a compostos húmicos nos não cultivados e as inorgânicas ligadas a Fe e Al nos cultivados.

\section{LITE RATURA CITADA}

BAHIA FILHO,A.F.C.;BRAGA, J .M.; RESENDE, M. \& RIBEIRO, A.C. Relação entre adsorção de fósforo e componentes mineralógicos da fração argila de Latossolos do Planalto Central. R. Bras. Ci. Solo, 7:221-226, 1983.
BIGHAM, J.M.; GOLDEN, D.C.; BUOL, S.W.; WEED, S.B. \& BOWEN, L.H. Iron oxide mineralogy of well-drained Ultisols and Oxisols: II. Influence on color, surface area, and phosphate retention. Soil Sci. Soc. Am. J., 42:825-830, 1978.

CHANG, S.C. \& JACKSON, M.L. Fractionation of soil phosphorous. Soil Sci., 84: 133-144, 1957.

CURI, N.; CAMARGO, O.A.; GUEDES, A. \& SILVEIRA, J.V. Sorção de fósforo em materiais de Latossolos do Brasil sudeste. In: REUNIÃO DE CLASSIFICAÇÃO, CORRELAÇÃO DE SOLOS E INTERPRETAÇÃO DA APTIDÃO AGRÍ COLA, 3., Rio de J aneiro, 1988. Anais. Rio de J aneiro, Empresa Brasileira de Pesquisa Agropecuária, Sociedade Brasileira de Ciência do Solo, 1988. p.267-282.

CURI, N. \& FRANZMEIER, D.P. Toposequence of Oxisols from the Central Plateau of Brazil. Soil Sci. Soc. Am. J., 48:341346, 1984.

CURI, N. \& FRANZMEIER, D.P. Effect of parent rocks on chemical and mineralogical properties of some Oxisols in Brazil. Soil Sci. Soc. Am. J ., 51:153-158, 1987.

EMPRESA BRASILEIRA DE PESQUISA AGROPECUÁRIA EMBRAPA. Centro Nacional de Pesquisa de Solos. Manual de métodos de análise do solo. Rio de J aneiro, 1997. 212p.

EMPRESA BRASILEIRA DE PESQUISA AGROPECUÁRIA EMBRAPA. Centro Nacional de Pesquisa de Solos. Sistema brasileiro de classificação de solos. Rio de J aneiro, 1999. 412p.

FROSSARD, E.; BROSSARD, M.; HEDLEY, M.J. \& METHERELL, A. Reactions controlling the cycling of $P$ in soils. I n:TIESSEN, H., ed. P ciding in terrestrial and aquatic ecosystem; a global perspective. New York, J ohn Wiley \& Sons, 1994. p.1-65.

GOEDERT, W.J . Solos dos cerrados - tecnol ogias e estratégias de manejo. São Paulo, Nobel, 1987. 422p.

GUIMARÃES, D. Contribuição ao estudo dos tufos vulcânicos da Mata da Corda. Belo Horizonte, I nstituto de Tecnologia Industrial, 1955. 31p. (Boletim, 18)

HEDLEY, M.J .; STEWARD, W.B. \& CHAUHAN, B.S. Changes in inorganic and organic soil phosphorus fractions induced by cultivation practices and laboratory incubation. Soil Sci. Soc. Am. J , 46:970-976, 1982. 
KÄMPF, N. \& SCHWERTMANN, U. The 5M-NaOH Concentration treatment for iron oxides in soils. Clays Clay Miner., 30:401-408, 1982.

KÄMPF, N.; RESENDE, M. \& CURI, N. I ron Oxides in Brazilian Oxisols. In: INTERNATIONAL SOIL CLASSIFICATION WORKSHOP, 8., Rio de J aneiro, 1988. Proceedings. Rio de J aneiro, Empresa Brasileira de Pesquisa Agropecuária, 1988. p.190-199.

KÄMPF, N. \& CURI, N. Óxidos deferro: indicadores de ambientes pedogênicos e geoquímicos. Tópicos Ci. Solo, 1:111-142, 2000.

KER, J.C. Mineralogia, sorção e dessorção de fosfato, magnetização e elementos traços de Latossolos do Brasil. Viçosa, Universidade Federal de Viçosa, 1995. 181p. (Tese de Doutorado)

LEAL, J .R. \& VELOSO, A.C.S. Adsorção de fosfato em Latossolo sob vegetação de cerrado. Pesq. Agropec. Bras., 8:81-88, 1973.

LEMOS, M.S.S; CURI, N.; MARQUES, J.J.G.S.M. \& ERNESTO SOBRINHO, F. Evaluation of characteristics of Cambisols derived from limestone in low tablelands in northeastern Brazil: I mplications for management. Pesq. Agropec. Bras., 32:825-834, 1997.

LINDSAY, W.L. Chemical equilibria in soils. New York:J ohn Wiley \& Sons, 1979. 449p.

LINQUIST, B.A.; SINGLETON, P.W.; YOST, R.S. \& CASSMAN, K.G. Aggregate size effects on the sorption and release of phosphorus in an Ultisol. Soil Sci. Soc. Am. J., 61:160-66, 1997.

MCLAUGHLIN, R.R.; RYDEN, J.C. \& SYERS, J.R. Sorption of inorganic phosphate by iron and aluminum containing components. J. Soil Sci., 32:365-377, 1981.

MALAVOLTA, E. Manual dequímica agrícola; nutrição de plantas e fertilidade do solo. São Paulo, Ceres, 1976. 528p.

MARQUES, J.J .G.S.M. Trace element distributions in Brazilian soils at thelandscape and micrometer scales. West Lafayette, Purdue University, 2000. 173p. (Tese de Doutorado)

MEHRA, O.P. \& J ACKSON, M.L. I ron oxide removal from soils and clays by dithionite-citrate system buffered with sodium bicarbonate. In: SWINEFORD, A.D.A., ed. CLAYS CLAY MINERALS CONFERENCE, 7., Washington, 1958. EImsdorf, Pergamon Press, 1960. p.317-327.

MESQUITA FILHO, M.V. \& TORRENT, J. Phosphate sorption as related to mineralogy of a hydrosequence of soils from the Cerrado region (Brazil). Geoderma, 58:107-123, 1993.

MOTTA, P.E.F. Fósforo em Latossolos com diferentes mineralogias, cultivados e não cultivados: fracionamento, sorção e disponibilidade para soja e braquiária com e sem micorriza. Lavras, Universidade Federal de Lavras, 1999. 158p. (Tese de Doutorado)

MOURA FILHO, W. \& BUOL, S.W. Studies of a Latosol Roxo (Eutrustox) in Brazil: Micromorphology effect on ion release. Experientiae, 21:161-177, 1976.
MURPHY, J. \& RILEY, J.P. A modified single solution method for the determination of phosphate in natural waters. Anal. Chim. Acta, 27:31-36, 1962.

OLSEN, S.R. \& WATANABE, F.S. A method to determine a phosphorous adsorption maximum of soils as measured by the Langmuir isotherm. Soil Sci. Soc. Am. Proc., 21:144149, 1957.

PARFITT, R.L. The availability of $P$ from phosphate-goethite brindging complexes, dessorption and uptake by ryegrass. Plant Soil, 53:55-65, 1979.

PAVAN, M .A. \& CHAVES, J.C.D. Alterações nas frações de fósforo no solo associadas com a densidade populacional de cafeeiros. R. Bras. Ci. Solo, 20:251-256, 1996.

PEÑA, F. \& TORRENT, J. Relationships between phosphate sorption and iron oxides in Alfisols from a river terrace sequence of Mediterranean Spain. Geoderma, 33:283-296, 1984.

RAIJ , B. van. Fertilidade do solo e adubação. Piracicaba, Ceres, 1991.343p.

RESENDE, M.; CURI, N.; REZENDE, S.B. \& CORRÊA, G.F. Pedologia: base para distinção de ambientes. Viçosa, NEPUT, 1999a. 367p.

RESENDE, M. Mineralogy, chemistry, morphology and geomorphology of some soils of the Central Plateau of Brazil. West Lafayette, PurdueUniversity, 1976. 237p. (Tese de Doutorado)

RUAN, H.D. \& GILKES, R.J . Kinetics of phosphate sorption and desorption by synthetic al uminous goethite before and after thermal transformation to hematite. Clay Miner., 31:6374, 1996.

SCHEFFER, F.; WESTLE, E. \& LUDWEIG, F. On the question of hydrated iron oxides in soil. Chemie der Erde, J ena, v.1819:51-64, 1958.

SCHULZE, D.G. The influence of aluminum on iron oxides. VII: unit cell dimensions of al substituted goethites and estimation of Al from them. Clays Clay Miner., 32:36-44, 1984.

SCHWERTMANN, U. Differenzienrung der Eisenoxide des Bödens durch Extraktion mit Ammoniumoxalat-lösung. Z. Pflanzenernahr. und Bodenkd., 105:105-202, 1964.

SILVA, C.A.; ANDERSON, S.J . \& GUILHERME, L.R.G. Uso de cromatografia de exclusão por tamanho na caracterização de substâncias húmicas de Latossolo Vermel ho-E scuro sob efeito de calagem. R. Bras.Ci. Solo, 24:495-503, 2000.

TIESSEN, H.; STEWART, J.W.B. \& MOIR, J .O. Changes in organic and inorganic phosphorus composition of two grassland soils and their particle size fractions during 60-90 years of cultivation. J. Soil Sci., 34:815-823, 1983.

TORRENT, J .; BARRON, V. \& SCHWERTMANN, U. Phosphate adsorption and desorption by goethites differing in crystal morphology. Soil Sci. Soc. Am. J ., 54:1007-1012, 1990.

VETTORI, L. Métodos de análise de solos. Rio de J aneiro, Ministério da Agricultura, 1969. 24p. (Boletim técnico, 7) 
P.E.F. MOTTA et al. 